\title{
INFORMATION, INFLATION, AND INTEREST
}

\author{
LANE P. HUGHSTON \\ Department of Mathematics, Imperial College London \\ London SW7 2AZ, UK \\ E-mail: lane.hughston@imperial.ac.uk \\ ANDREA MACRINA \\ Department of Mathematics, King's College London \\ The Strand, London WC2R 2LS, UK \\ E-mail: andrea.macrina@kcl.ac.uk
}

\begin{abstract}
We propose a class of discrete-time stochastic models for the pricing of inflationlinked assets. The paper begins with an axiomatic scheme for asset pricing and interest rate theory in a discrete-time setting. The first axiom introduces a "risk-free" asset, and the second axiom determines the intertemporal pricing relations that hold for dividend-paying assets. The nominal and real pricing kernels, in terms of which the price index can be expressed, are then modelled by introducing a Sidrauski-type utility function depending on (a) the aggregate rate of consumption, and (b) the aggregate rate of real liquidity benefit conferred by the money supply. Consumption and money supply policies are chosen such that the expected joint utility obtained over a specified time horizon is maximised subject to a budget constraint that takes into account the "value" of the liquidity benefit associated with the money supply. For any choice of the bivariate utility function, the resulting model determines a relation between the rate of consumption, the price level, and the money supply. The model also produces explicit expressions for the real and nominal pricing kernels, and hence establishes a basis for the valuation of inflation-linked securities.
\end{abstract}

I. Introduction. In this paper we apply the information-based asset pricing scheme proposed in Brody et al. 2007, 2008, and Macrina 2006, to introduce a class of discretetime models for interest rates and inflation. The key idea is that market participants have at any time partial information about the future values of the macro-economic

2000 Mathematics Subject Classification: 91B28, 91B44, 91B64.

Key words and phrases: inflation, interest rate models, partial information, price level, money supply, consumption, liquidity, utility, transversality condition.

The paper is in final form and no version of it will be published elsewhere. 
factors that determine interest rates and price levels. We present a model for such partial information, and show how it leads to a novel framework for the arbitrage-free dynamics of real and nominal interest rates, price-indices, and index-linked securities.

We begin with a general model for discrete-time asset pricing. We take a pricing kernel approach, which builds in the arbitrage-free property and provides the desired link to economic equilibrium. We require that the pricing kernel should be consistent with a pair of axioms, one giving the intertemporal relations for dividend-paying assets, and the other relating to the existence of a money market asset. Instead of directly assuming the existence of a previsible money-market account, we make a weaker assumption, namely the existence of an asset that offers a positive rate of return. It can be deduced, however, that the existence of a positive-return asset is sufficient to imply the existence of a previsible money-market account, once the intertemporal relations implicit in the first axiom are taken into account. The main result of Section II is the derivation of a general expression for the price process of a limited-liability asset. This expression includes two terms, one being the discounted and risk-adjusted value of the dividend stream, and the other characterising retained earnings. The vanishing of the latter is shown in Proposition 1 to be given by a transversality condition, equation (9). In particular, we are able to show (under the conditions of Axioms A and B) that in the case of a limited-liability asset with no permanently retained earnings, the general form of the price process is given by the ratio of a potential and the pricing kernel, as expressed in equation (20). In Section III we consider the per-period rate of return $\left\{\bar{r}_{i}\right\}$ offered by the positive return asset, and we show in Proposition 2 that there exists a constant-value asset with limited liability such that the associated dividend flow is given by $\left\{\bar{r}_{i}\right\}$. This result is then used in Proposition 3 to establish that the pricing kernel admits a decomposition of the form (31). In Proposition 4 we prove a converse to this result, thus giving a procedure for constructing examples of systems satisfying Axioms A and B. The method involves the introduction of an increasing sequence that converges to an integrable random variable. Given the sequence we construct an associated pricing kernel and positive-return asset satisfying the intertemporal relations.

In Section IV we introduce the nominal discount bond system arising with the specification of the pricing kernel, and in Proposition 5 we show that the discount bond system admits a representation of the Flesaker-Hughston type. In Section V we consider the case when the positive-return asset has a previsible price process, and hence can be interpreted (in a standard way) as a money-market account, or "risk-free" asset. The results of the previous sections do not depend on this additional assumption. A previsible money-market account has the structure of a series of one-period discount-bond investments. Then in Proposition 6 we show under the conditions of Axioms A and B that there exists a unique previsible money-market account. In other words, although we only assume the existence of a positive-return asset, we can establish the existence of a money-market account.

In Section VI we outline a general approach to interest rate modelling in the information-based framework, in a discrete-time setting. In Section VII we then propose a class of models for the pricing of inflation-linked assets. The nominal and real pricing kernels, in terms of which the consumer price index can be expressed, are modelled by introducing a 
bivariate utility function depending on (a) consumption, and (b) the real liquidity benefit conferred by the money supply. Consumption and money supply policies are chosen such that the expected joint utility obtained over a specified time horizon is maximised, subject to a budget constraint that takes into account the "value" of the liquidity benefit associated with the money supply. For any choice of the bivariate utility function, the resulting model determines a relation between the rate of consumption, the price level, and the money supply. The model produces explicit expressions for the real and nominal pricing kernels, and hence establishes a basis for the valuation of inflation-linked securities.

II. Asset pricing in discrete time. The development of asset-pricing theory in discrete time has been pursued by many authors. In the context of interest rate modelling, it is worth recalling that the first example of a fully developed term-structure model where the initial discount function is freely specifiable is that of Ho \& Lee 1986, in a discrete-time setting.

For our purposes it will be useful to develop a general discrete-time scheme from first principles, taking an axiomatic approach in the spirit of Hughston \& Rafailidis (2005). Let $\left\{t_{i}\right\}_{i=0,1,2, \ldots}$ denote a sequence of discrete times, where $t_{0}$ represents the present and $t_{i+1}>t_{i}$ for all $i \in \mathbb{N}_{0}$. We assume that the sequence $\left\{t_{i}\right\}$ is unbounded in the sense that for any given time $T$ there exists a value of $i$ such that $t_{i}>T$. We do not assume that the elements of $\left\{t_{i}\right\}$ are equally spaced; for some applications, however, we can consider the case where $t_{n}=n \tau$ for all $n \in \mathbb{N}_{0}$ and for some unit of time $\tau$.

Each asset is characterised by a pair of processes $\left\{S_{t_{i}}\right\}_{i \geq 0}$ and $\left\{D_{t_{i}}\right\}_{i \geq 0}$ which we refer to as the "value process" and the "dividend process", respectively. We interpret $D_{t_{i}}$ as a random cash flow or dividend paid to the owner of the asset at time $t_{i}$. Then $S_{t_{i}}$ denotes the "ex-dividend" value of the asset at $t_{i}$. We can think of $S_{t_{i}}$ as the cash flow that would result if the owner were to dispose of the asset at time $t_{i}$.

For simplicity, we shall frequently use an abbreviated notation, and write $S_{i}=S_{t_{i}}$ and $D_{i}=D_{t_{i}}$. Thus $S_{i}$ denotes the value of the asset at time $t_{i}$, and $D_{i}$ denotes the dividend paid at time $t_{i}$. Both $S_{i}$ and $D_{i}$ are expressed in nominal terms, i.e. in units of a fixed base currency. We use the term "asset" in the broad sense here - the scheme is thus applicable to any liquid financial position for which the values and cash flows are well defined, and for which the principles of no arbitrage are applicable.

The unfolding of random events in the economy will be represented with the specification of a probability space $(\Omega, \mathcal{F}, \mathbb{P})$ equipped with a filtration $\left\{\mathcal{F}_{i}\right\}_{i \geq 0}$ which we call the "market filtration". For the moment we regard the market filtration as given, but later we shall construct it explicitly. For each asset we assume that the associated value and dividend processes are adapted to $\left\{\mathcal{F}_{i}\right\}$. In what follows $\mathbb{P}$ is taken to be the "physical" or "objective" probability measure; all equalities and inequalities between random variables are to be understood as holding almost surely with respect to $\mathbb{P}$. For convenience we often write $\mathbb{E}_{i}[-]$ for $\mathbb{E}\left[-\mid \mathcal{F}_{i}\right]$.

In order to ensure the absence of arbitrage in the financial markets and to establish intertemporal pricing relations, we assume the existence of a strictly positive pricing kernel $\left\{\pi_{i}\right\}_{i \geq 0}$, and make the following assumptions: 
Axiom A. For any asset with associated value process $\left\{S_{i}\right\}_{i \geq 0}$ and dividend process $\left\{D_{i}\right\}_{i \geq 0}$, the process $\left\{M_{i}\right\}_{i \geq 0}$ defined by

$$
M_{i}=\pi_{i} S_{i}+\sum_{n=0}^{i} \pi_{n} D_{n}
$$

is a martingale, i.e. $\mathbb{E}\left[\left|M_{i}\right|\right]<\infty$ for all $i \in \mathbb{N}_{0}$, and $\mathbb{E}\left[M_{j} \mid \mathcal{F}_{i}\right]=M_{i}$ for all $i \leq j$.

Axiom B. There exists a strictly positive non-dividend-paying asset, with value process $\left\{\bar{B}_{i}\right\}_{i \geq 0}$, that offers a strictly positive return, i.e. such that $\bar{B}_{i+1}>\bar{B}_{i}$ for all $i \in \mathbb{N}_{0}$. We assume that the process $\left\{\bar{B}_{i}\right\}$ is unbounded in the sense that for any $b \in \mathbb{R}$ there exists a time $t_{i}$ such that $\bar{B}_{i}>b$.

Given this axiomatic scheme, we proceed to explore its consequences. We shall see that Axioms A and B lead to a relation between the price of an asset and the associated dividend stream. This relation appears in Proposition 1. The notation $\left\{\bar{B}_{i}\right\}$ is used in Axiom $\mathrm{B}$ to distinguish the positive return asset from the previsible money-market account asset $\left\{B_{i}\right\}$ that will be introduced later; in particular, in Proposition 6 it will be shown that Axioms A and B imply the existence of a unique money-market account asset. We note that since the positive-return asset is non-dividend paying, it follows from Axiom A that $\left\{\pi_{i} \bar{B}_{i}\right\}$ is a martingale. Writing $\bar{\rho}_{i}=\pi_{i} \bar{B}_{i}$, we have $\pi_{i}=\bar{\rho}_{i} / \bar{B}_{i}$. Since $\left\{\bar{B}_{i}\right\}$ is assumed to be strictly increasing, we see that $\left\{\pi_{i}\right\}$ is a supermartingale. In fact, we have the strict inequality

$$
\mathbb{E}_{i}\left[\pi_{j}\right]=\mathbb{E}_{i}\left[\frac{\bar{\rho}_{j}}{\bar{B}_{j}}\right]<E_{i}\left[\frac{\bar{\rho}_{j}}{\bar{B}_{i}}\right]=\frac{E_{i}\left[\bar{\rho}_{j}\right]}{\bar{B}_{i}}=\pi_{i} .
$$

The significance of $\left\{\bar{\rho}_{i}\right\}$ is that it has the interpretation of being the likelihood ratio appropriate for a change of measure from the objective measure $\mathbb{P}$ to the equivalent martingale measure $\mathbb{Q}$ characterised by the property that non-dividend-paying assets when expressed in units of the numeraire $\left\{\bar{B}_{i}\right\}$ are martingales.

We recall the definition of a potential. An adapted process $\left\{x_{i}\right\}_{0 \leq i<\infty}$ on a probability space $(\Omega, \mathcal{F}, \mathbb{P})$ with filtration $\left\{\mathcal{F}_{i}\right\}$ is said to be a potential if $\left\{x_{i}\right\}$ is a non-negative supermartingale and $\lim _{i \rightarrow \infty} \mathbb{E}\left[x_{i}\right]=0$. It is straightforward to show that $\left\{\pi_{i}\right\}$ is a potential. We need to demonstrate that given any $\epsilon>0$ we can find a time $t_{j}$ such $\mathbb{E}\left[\pi_{n}\right]<\epsilon$ for all $n \geq j$. This follows from the assumption that the positive-return asset price process $\left\{\bar{B}_{i}\right\}$ is unbounded in the sense specified in Axiom B. Thus given $\epsilon$ let us set $b=\bar{\rho}_{0} / \epsilon$. Now given $b$ we can find a time $t_{j}$ such that $\bar{B}_{t_{n}}>b$ for all $n \geq j$. But for that value of $t_{j}$ we have $\mathbb{E}\left[\pi_{j}\right]=\mathbb{E}\left[\bar{\rho}_{j} / \bar{B}_{j}\right]<\mathbb{E}\left[\bar{\rho}_{j}\right] / b=\epsilon$, and hence $\mathbb{E}\left[\pi_{n}\right]<\epsilon$ for all $n \geq j$. It follows that

$$
\lim _{i \rightarrow \infty} \mathbb{E}\left[\pi_{i}\right]=0
$$

Next we recall the Doob decomposition for discrete-time supermartingales (see, e.g., Meyer 1966 , chapter 7$)$. If $\left\{x_{i}\right\}$ is a supermartingale on a probability space $(\Omega, \mathcal{F}, \mathbb{P})$ with filtration $\left\{\mathcal{F}_{i}\right\}$, then there exists a martingale $\left\{y_{i}\right\}$ and a previsible increasing process $\left\{a_{i}\right\}$ such that $x_{i}=y_{i}-a_{i}$ for all $i \geq 0$. By previsible, we mean that $a_{i}$ is $\mathcal{F}_{i-1}$-measurable. The decomposition is given explicitly by $a_{0}=0$ and $a_{i}=a_{i-1}+x_{i-1}-\mathbb{E}_{i-1}\left[x_{i}\right]$ for $i \geq 1$. 
It follows that the pricing kernel admits a decomposition of the form

$$
\pi_{i}=Y_{i}-A_{i}
$$

where $A_{0}=0$ and

$$
A_{i}=\sum_{n=0}^{i-1}\left(\pi_{n}-\mathbb{E}_{n}\left[\pi_{n+1}\right]\right)
$$

for $i \geq 1 ;$ and where $Y_{0}=\pi_{0}$ and

$$
Y_{i}=\sum_{n=0}^{i-1}\left(\pi_{n+1}-\mathbb{E}_{n}\left[\pi_{n+1}\right]\right)+\pi_{0}
$$

for $i \geq 1$. The Doob decomposition for $\left\{\pi_{i}\right\}$ has an interesting expression in terms of discount bonds, which we shall mention later, in Section V.

In the case of a potential $\left\{x_{i}\right\}$ it can be shown (see Gihman \& Skorohod 1979) that $a_{\infty}=\lim _{i \rightarrow \infty} a_{i}$ exists, and that $x_{i}=\mathbb{E}_{i}\left[a_{\infty}\right]-a_{i}$. As a consequence, we conclude that the pricing kernel admits a decomposition of the form

$$
\pi_{i}=\mathbb{E}_{i}\left[A_{\infty}\right]-A_{i}
$$

where $\left\{A_{i}\right\}$ is the previsible process defined by (5). With these facts in hand, we shall establish a useful result concerning the pricing of limited-liability assets. By a limitedliability asset we mean an asset such that $S_{i} \geq 0$ and $D_{i} \geq 0$ for all $i \in \mathbb{N}$.

Proposition 1. Let $\left\{S_{i}\right\}_{i \geq 0}$ and $\left\{D_{i}\right\}_{i \geq 0}$ be the value and dividend processes associated with a limited-liability asset. Then $\left\{S_{i}\right\}$ is of the form

$$
S_{i}=\frac{m_{i}}{\pi_{i}}+\frac{1}{\pi_{i}} \mathbb{E}_{i}\left[\sum_{n=i+1}^{\infty} \pi_{n} D_{n}\right],
$$

where $\left\{m_{i}\right\}$ is a non-negative martingale that vanishes if and only if the following transversality condition holds:

$$
\lim _{j \rightarrow \infty} \mathbb{E}\left[\pi_{j} S_{j}\right]=0
$$

Proof. It follows from Axiom A, as a consequence of the martingale property, that

$$
\pi_{i} S_{i}+\sum_{n=0}^{i} \pi_{n} D_{n}=\mathbb{E}_{i}\left[\pi_{j} S_{j}+\sum_{n=0}^{j} \pi_{n} D_{n}\right]
$$

for $i \leq j$. Taking the limit $j \rightarrow \infty$ on the right-hand side of this relation we have

$$
\pi_{i} S_{i}+\sum_{n=0}^{i} \pi_{n} D_{n}=\lim _{j \rightarrow \infty} \mathbb{E}_{i}\left[\pi_{j} S_{j}\right]+\lim _{j \rightarrow \infty} \mathbb{E}_{i}\left[\sum_{n=0}^{j} \pi_{n} D_{n}\right] .
$$

Since $\pi_{i} D_{i} \geq 0$ for $i \in \mathbb{N}_{0}$, it follows from the conditional form of the monotone convergence theorem - see, e.g., Steele 2001, Williams 1991 - that

$$
\lim _{j \rightarrow \infty} \mathbb{E}_{i}\left[\sum_{n=0}^{j} \pi_{n} D_{n}\right]=\mathbb{E}_{i}\left[\lim _{j \rightarrow \infty} \sum_{n=0}^{j} \pi_{n} D_{n}\right],
$$


and hence that

$$
\pi_{i} S_{i}+\sum_{n=0}^{i} \pi_{n} D_{n}=\lim _{j \rightarrow \infty} \mathbb{E}_{i}\left[\pi_{j} S_{j}\right]+\mathbb{E}_{i}\left[\sum_{n=0}^{\infty} \pi_{n} D_{n}\right] .
$$

Now let us define

$$
m_{i}=\lim _{j \rightarrow \infty} \mathbb{E}_{i}\left[\pi_{j} S_{j}\right]
$$

Then clearly $m_{i} \geq 0$ for all $i \in \mathbb{N}_{0}$. We see, moreover, that $\left\{m_{i}\right\}_{i \geq 0}$ is a martingale, since $m_{i}=M_{i}-\mathbb{E}_{i}\left[F_{\infty}\right]$, where $M_{i}$ is defined as in equation (1), and

$$
F_{\infty}=\sum_{n=0}^{\infty} \pi_{n} D_{n}
$$

It is implicit in the axiomatic scheme that the sum $\sum_{n=0}^{\infty} \pi_{n} D_{n}$ converges in the case of a limited-liability asset. This follows as a consequence of the martingale convergence theorem and Axiom A. Thus, writing equation (13) in the form

$$
\pi_{i} S_{i}+\sum_{n=0}^{i} \pi_{n} D_{n}=m_{i}+\mathbb{E}_{i}\left[\sum_{n=0}^{\infty} \pi_{n} D_{n}\right],
$$

after some re-arrangement of terms we obtain

$$
\pi_{i} S_{i}=m_{i}+\mathbb{E}_{i}\left[\sum_{n=i+1}^{\infty} \pi_{n} D_{n}\right]
$$

and hence (8), as required. On the other hand, by the martingale property of $\left\{m_{i}\right\}$ we have $\mathbb{E}\left[m_{i}\right]=m_{0}$ and hence

$$
\mathbb{E}\left[m_{i}\right]=\lim _{j \rightarrow \infty} \mathbb{E}\left[\pi_{j} S_{j}\right]
$$

for all $i \in \mathbb{N}$. Thus since $m_{i} \geq 0$ we see that (9) holds if and only if $\left\{m_{i}\right\}=0$.

The interpretation of the transversality condition is as follows. For each $j \in \mathbb{N}_{0}$ the expectation $V_{j}=\mathbb{E}\left[\pi_{j} S_{j}\right]$ measures the present value of an instrument that pays at $t_{j}$ an amount equal to the proceeds of a liquidation of the asset with price process $\left\{S_{i}\right\}_{i \geq 0}$. If $\lim _{j \rightarrow \infty} V_{j}=0$ then one can say that in the long term all of the value of the asset will be dispersed in its dividends. On the other hand, if some or all of the dividends are "retained" indefinitely, then $\left\{V_{j}\right\}$ will retain some value, even in the limit as $t_{j}$ goes to infinity. The relation $m_{i}=M_{i}-\mathbb{E}_{i}\left[F_{\infty}\right]$ shows that $\left\{m_{i}\right\}$ represents that part of the martingale $\left\{M_{i}\right\}$ that is not accounted for by the dividend flow.

The following example may clarify this interpretation. Suppose investors put $\$ 100 \mathrm{~m}$ of capital into a new company. The management of the company deposit $\$ 10 \mathrm{~m}$ into a money market account. The remaining $\$ 90 \mathrm{~m}$ is invested in a risky line of business, the proceeds of which, after costs, are paid to share-holders as dividends. At time $t_{i}$ we have $S_{i}=B_{i}+H_{i}$, where $B_{i}$ is a position in the money market account initialised at $\$ 10 \mathrm{~m}$, and $H_{i}$ is the value of the remaining dividend flow. Now $\left\{\pi_{i} B_{i}\right\}$ is a martingale, and thus $\mathbb{E}\left[\pi_{i} B_{i}\right]=\$ 10 \mathrm{~m}$ for all $i \in \mathbb{N}_{0}$, and hence $\lim _{i \rightarrow \infty} \mathbb{E}\left[\pi_{i} B_{i}\right]=\$ 10 \mathrm{~m}$. On the other hand $\lim _{i \rightarrow \infty} \mathbb{E}\left[\pi_{i} H_{i}\right]=0$; this means that given any value $h$ we can find a time $T$ such that $\mathbb{E}\left[\pi_{i} H_{i}\right]<h$ for all $t_{i} \geq T$. 
There are other ways of "retaining" funds than putting them into a domestic money market account. For example, one could put the $\$ 10 \mathrm{~m}$ into a foreign bank account; or one could invest it in shares in a securities account, with a standing order that dividends should be immediately re-invested in further shares. If the investment is in a general "dividend-retaining" asset (such as a foreign bank account), then $\left\{m_{i}\right\}$ can be any nonnegative martingale. The content of Proposition 1 is that a limited-liability investment can be separated in a unique way into a "pure growth" component and a "pure dividend" component.

In the case of a "pure dividend" investment, i.e. in an asset for which the transversality condition is satisfied, the price is directly related to the future dividend flow, and we have

$$
S_{i}=\frac{1}{\pi_{i}} \mathbb{E}_{i}\left[\sum_{n=i+1}^{\infty} \pi_{n} D_{n}\right] .
$$

This is the so-called "fundamental equation" which some authors have used directly as a basis for asset pricing theory - see, e.g., Cochrane 2005. One could in principle take the point of view that even in the case of a pure growth asset such as a money market account a dividend is eventually paid (for example, when one closes down the account). In that case, all assets can be regarded as "pure dividend" assets. But it is convenient (and standard) to maintain the notion of "pure growth" assets in finance theory, even if this involves an element of abstraction. An alternative way of writing (19) is in the form

$$
S_{i}=\frac{1}{\pi_{i}}\left(\mathbb{E}_{i}\left[F_{\infty}\right]-F_{i}\right)
$$

where

$$
F_{i}=\sum_{n=0}^{i} \pi_{n} D_{n}, \quad \text { and } \quad F_{\infty}=\lim _{i \rightarrow \infty} F_{i} .
$$

It is straightforward to show that the process $\left\{\pi_{i} S_{i}\right\}$ is a potential. Clearly, $\left\{\mathbb{E}_{i}\left[F_{\infty}\right]-\right.$ $\left.F_{i}\right\}$ is a positive supermartingale, since $\left\{F_{i}\right\}$ is increasing; and by the tower property and the monotone convergence theorem we have $\lim _{i \rightarrow \infty} \mathbb{E}\left[\mathbb{E}_{i}\left[F_{\infty}\right]-F_{i}\right]=\mathbb{E}\left[F_{\infty}\right]-$ $\lim _{i \rightarrow \infty} \mathbb{E}\left[F_{i}\right]=\mathbb{E}\left[F_{\infty}\right]-\mathbb{E}\left[\lim _{i \rightarrow \infty} F_{i}\right]=0$. On the other hand, $\left\{\pi_{i}\right\}$ is also a potential, so we conclude that in the case of a pure dividend asset the price process can be expressed as a ratio of potentials, thus giving us a discrete-time analogue of a result obtained by Rogers 1997. Indeed, the role of the concept of a potential as it appears here is consistent with the continuous-time theories developed by Flesaker \& Hughston 1996, Rogers 1997, Rutkowski 1997, Jin \& Glasserman 2001, Hughston \& Rafailidis 2005, and others, where similar structures arise.

III. Nominal pricing kernel and nominal interest rates. To proceed further we need to say more about the relation between the pricing kernel $\left\{\pi_{i}\right\}$ and the positivereturn asset $\left\{\bar{B}_{i}\right\}$. To this end let us write

$$
\bar{r}_{i}=\frac{\bar{B}_{i}-\bar{B}_{i-1}}{\bar{B}_{i-1}}
$$

for the rate of return on the positive-return asset realised at time $t_{i}$ on an investment made at time $t_{i-1}$. Since the time interval $t_{i}-t_{i-1}$ is not necessarily small, there is no 
specific reason to presume that the rate of return $\bar{r}_{i}$ is already known at time $t_{i-1}$. This is consistent with the fact that we have assumed that $\left\{\bar{B}_{i}\right\}$ is $\left\{\mathcal{F}_{i}\right\}$-adapted. The notation $\bar{r}_{i}$ is used here to distinguish the rate of return on the positive-return asset from the rate of return $r_{i}$ on the money market account, which will be introduced in Section V.

Next we present a simple argument to motivate the idea that there should exist an asset with constant value unity that pays a dividend stream given by $\left\{\bar{r}_{i}\right\}$. We consider the following portfolio strategy. The portfolio consists at any time of a certain number of units of the positive-return asset. Let $\phi_{i}$ denote the number of units, so that at time $t_{i}$ the (ex-dividend) value of the portfolio is given by $V_{i}=\phi_{i} \bar{B}_{i}$. Then in order to have $V_{i}=1$ for all $i \geq 0$ we set $\phi_{i}=1 / \bar{B}_{i}$. Let $D_{i}$ denote the dividend paid out by the portfolio at time $t_{i}$. Then clearly if the portfolio value is to remain constant we must have $D_{i}=\phi_{i-1} \bar{B}_{i}-\phi_{i-1} \bar{B}_{i-1}$ for all $i \geq 1$. It follows immediately that $D_{i}=\bar{r}_{i}$, where $\bar{r}_{i}$ is given by $(22)$.

This shows that we can construct a portfolio with a constant value and with the desired cash flows. Now we need to show that such a system satisfies Axiom A.

Proposition 2. There exists an asset with constant nominal value $S_{i}=1$ for all $i \in \mathbb{N}_{0}$, for which the associated cash flows are given by $\left\{\bar{r}_{i}\right\}_{i \geq 1}$.

Proof. We need to verify that the conditions of Axiom A are satisfied in the case for which $S_{i}=1$ and $D_{i}=\bar{r}_{i}$ for $i \in \mathbb{N}_{0}$. In other words we need to show that

$$
\pi_{i}=\mathbb{E}_{i}\left[\pi_{j}\right]+\mathbb{E}_{i}\left[\sum_{n=i+1}^{j} \pi_{n} \bar{r}_{n}\right]
$$

for $i \leq j$. The calculation proceeds as follows. We observe that

$$
\begin{aligned}
\mathbb{E}_{i}\left[\sum_{n=i+1}^{j} \pi_{n} \bar{r}_{n}\right] & =\mathbb{E}_{i}\left[\sum_{n=i+1}^{j} \pi_{n} \frac{\bar{B}_{n}-\bar{B}_{n-1}}{\bar{B}_{n-1}}\right]=\mathbb{E}_{i}\left[\sum_{n=i+1}^{j}\left(\frac{\bar{\rho}_{n}}{\bar{B}_{n-1}}-\frac{\bar{\rho}_{n}}{\bar{B}_{n}}\right)\right] \\
& =\mathbb{E}_{i}\left[\sum_{n=i+1}^{j}\left(\mathbb{E}_{n-1}\left[\frac{\bar{\rho}_{n}}{\bar{B}_{n-1}}\right]-\frac{\bar{\rho}_{n}}{\bar{B}_{n}}\right)\right],
\end{aligned}
$$

the last step being achieved by use of the tower property. It follows then by use of the martingale property of $\left\{\rho_{n}\right\}$ that

$$
\begin{aligned}
\mathbb{E}_{i}\left[\sum_{n=i+1}^{j} \pi_{n} \bar{r}_{n}\right] & =\mathbb{E}_{i}\left[\sum_{n=i+1}^{j}\left(\frac{1}{\bar{B}_{n-1}} \mathbb{E}_{n-1}\left[\bar{\rho}_{n}\right]-\frac{\bar{\rho}_{n}}{\bar{B}_{n}}\right)\right] \\
& =\mathbb{E}_{i}\left[\sum_{n=i+1}^{j}\left(\frac{\bar{\rho}_{n-1}}{\bar{B}_{n-1}}-\frac{\bar{\rho}_{n}}{\bar{B}_{n}}\right)\right] \\
& =\mathbb{E}_{i}\left[\frac{\bar{\rho}_{i}}{\bar{B}_{i}}\right]-\mathbb{E}_{i}\left[\frac{\bar{\rho}_{j}}{\bar{B}_{j}}\right]=\pi_{i}-\mathbb{E}_{i}\left[\pi_{j}\right]
\end{aligned}
$$

But that gives (23).

The existence of the constant-value asset leads to an alternative decomposition of the pricing kernel, which can be described as follows. 
Proposition 3. Let $\left\{\bar{B}_{i}\right\}$ be a positive-return asset satisfying the conditions of Axiom $B$, and let $\left\{\bar{r}_{i}\right\}$ be its rate-of-return process. Then the pricing kernel can be expressed in the form $\pi_{i}=\mathbb{E}_{i}\left[G_{\infty}\right]-G_{i}$, where $G_{i}=\sum_{n=1}^{i} \pi_{n} \bar{r}_{n}$ and $G_{\infty}=\lim _{i \rightarrow \infty} G_{i}$.

Proof. First we remark that if an asset has constant value then it satisfies the transversality condition (9). In particular, letting the constant be unity, we see that the transversality condition reduces to

$$
\lim _{i \rightarrow \infty} \mathbb{E}\left[\pi_{i}\right]=0,
$$

which is satisfied since $\left\{\pi_{i}\right\}$ is a potential. Next we show that

$$
\lim _{j \rightarrow \infty} \mathbb{E}_{i}\left[\pi_{j}\right]=0
$$

for all $i \in \mathbb{N}_{0}$. In particular, fixing $i$, we have $\mathbb{E}\left[\mathbb{E}_{i}\left[\pi_{j}\right]\right]=\mathbb{E}\left[\pi_{j}\right]$ by the tower property, and thus

$$
\lim _{j \rightarrow \infty} \mathbb{E}\left[\mathbb{E}_{i}\left[\pi_{j}\right]\right]=0
$$

by virtue of (26). But $\mathbb{E}_{i}\left[\pi_{j}\right]<\pi_{i}$ for all $j>i$, and $\mathbb{E}\left[\pi_{i}\right]<\infty$; hence by the dominated convergence theorem we have

$$
\lim _{j \rightarrow \infty} \mathbb{E}\left[\mathbb{E}_{i}\left[\pi_{j}\right]\right]=\mathbb{E}\left[\lim _{j \rightarrow \infty} \mathbb{E}_{i}\left[\pi_{j}\right]\right],
$$

from which we obtain (27), since the argument of the expectation is non-negative. As a consequence of (27) it follows from (23) that

$$
\pi_{i}=\lim _{j \rightarrow \infty} \mathbb{E}_{i}\left[\sum_{n=i+1}^{j} \pi_{n} \bar{r}_{n}\right]
$$

and thus by the monotone convergence theorem we have

$$
\pi_{i}=\mathbb{E}_{i}\left[\sum_{n=i+1}^{\infty} \pi_{n} \bar{r}_{n}\right]=\mathbb{E}_{i}\left[\sum_{n=1}^{\infty} \pi_{n} \bar{r}_{n}\right]-\sum_{n=1}^{i} \pi_{n} \bar{r}_{n}=\mathbb{E}_{i}\left[G_{\infty}\right]-G_{i},
$$

and that gives us the result of the proposition.

We shall establish a converse to this result, which allows us to construct a system satisfying Axioms A and B from any strictly-increasing non-negative adapted process that converges, providing a certain integrability condition holds.

Proposition 4. Let $\left\{G_{i}\right\}_{i \geq 0}$ be a strictly increasing adapted process satisfying $G_{0}=0$, and $\mathbb{E}\left[G_{\infty}\right]<\infty$, where $G_{\infty}=\lim _{i \rightarrow \infty} G_{i}$. Let the processes $\left\{\pi_{i}\right\},\left\{\bar{r}_{i}\right\}$, and $\left\{\bar{B}_{i}\right\}$, be defined by $\pi_{i}=\mathbb{E}_{i}\left[G_{\infty}\right]-G_{i}$ for $i \geq 0 ; \bar{r}_{i}=\left(G_{i}-G_{i-1}\right) / \pi_{i}$ for $i \geq 1 ; \bar{B}_{i}=\prod_{n=1}^{i}\left(1+\bar{r}_{n}\right)$ for $i \geq 1$, with $\bar{B}_{0}=1$. Let the process $\left\{\bar{\rho}_{i}\right\}$ be defined by $\bar{\rho}_{i}=\pi_{i} \bar{B}_{i}$ for $i \geq 0$. Then $\left\{\bar{\rho}_{i}\right\}$ is a martingale, and $\lim _{j \rightarrow \infty} \bar{B}_{j}=\infty$. Thus $\left\{\pi_{i}\right\}$ and $\left\{\bar{B}_{i}\right\}$, as constructed, satisfy Axioms $A$ and $B$.

Proof. Writing $g_{i}=G_{i}-G_{i-1}$ for $i \geq 1$ we have

$$
\pi_{i}=\mathbb{E}_{i}\left[G_{\infty}\right]-G_{i}=\mathbb{E}_{i}\left[\sum_{n=i+1}^{\infty} g_{n}\right]
$$


and

$$
\bar{B}_{i}=\prod_{n=1}^{i}\left(1+\bar{r}_{n}\right)=\prod_{n=1}^{i}\left(1+\frac{g_{n}}{\pi_{n}}\right)=\prod_{n=1}^{i}\left(\frac{\pi_{n}+g_{n}}{\pi_{n}}\right) .
$$

Hence, writing $\bar{\rho}_{i}=\pi_{i} \bar{B}_{i}$, we have

$$
\bar{\rho}_{i}=\pi_{i} \prod_{n=1}^{i}\left(\frac{\pi_{n}+g_{n}}{\pi_{n}}\right)=\left(\pi_{i}+g_{i}\right) \prod_{n=1}^{i-1}\left(\frac{\pi_{n}+g_{n}}{\pi_{n}}\right),
$$

and thus

$$
\bar{\rho}_{i}=\left(\pi_{i}+g_{i}\right) \bar{B}_{i-1}=\frac{\pi_{i}+g_{i}}{\pi_{i-1}} \bar{\rho}_{i-1} .
$$

To show that $\left\{\bar{\rho}_{i}\right\}$ is a martingale it suffices to verify for all $i \geq 1$ that $\mathbb{E}\left[\bar{\rho}_{i}\right]<\infty$ and that $\mathbb{E}_{i-1}\left[\bar{\rho}_{i}\right]=\bar{\rho}_{i-1}$. In particular, if $\mathbb{E}\left[\bar{\rho}_{i}\right]<\infty$ then the "take out what is known rule" applies, and by (32) and (34) we have

$$
\begin{aligned}
\mathbb{E}_{i-1}\left[\bar{\rho}_{i}\right] & =\mathbb{E}_{i-1}\left[\frac{\pi_{i}+g_{i}}{\pi_{i-1}} \bar{\rho}_{i-1}\right]=\frac{\bar{\rho}_{i-1}}{\pi_{i-1}} \mathbb{E}_{i-1}\left[\pi_{i}+g_{i}\right]=\frac{\bar{\rho}_{i-1}}{\pi_{i-1}} \mathbb{E}_{i-1}\left[\sum_{n=i}^{\infty} g_{n}\right] \\
& =\frac{\bar{\rho}_{i-1}}{\pi_{i-1}}\left(\mathbb{E}_{i-1}\left[G_{\infty}\right]-G_{i-1}\right)=\bar{\rho}_{i-1} .
\end{aligned}
$$

Here, in going from the first to the second line we have used the fact that $\mathbb{E}\left[\pi_{i}+g_{i}\right]<\infty$, together with the assumption that $\mathbb{E}\left[\bar{\rho}_{i}\right]<\infty$. To verify that $\mathbb{E}\left[\bar{\rho}_{i}\right]<\infty$ let us write

$$
J_{i-1}^{\alpha}=\min \left[\frac{\bar{\rho}_{i-1}}{\pi_{i-1}}, \alpha\right]
$$

for $\alpha \in \mathbb{N}_{0}$. Then by use of monotone convergence and the tower property we have

$$
\begin{aligned}
\mathbb{E}\left[\bar{\rho}_{i}\right] & =\mathbb{E}\left[\left(\pi_{i}+g_{i}\right) \lim _{\alpha \rightarrow \infty} J_{i-1}^{\alpha}\right]=\lim _{\alpha \rightarrow \infty} \mathbb{E}\left[\left(\pi_{i}+g_{i}\right) J_{i-1}^{\alpha}\right] \\
& =\lim _{\alpha \rightarrow \infty} \mathbb{E}\left[\mathbb{E}_{i-1}\left[\left(\pi_{i}+g_{i}\right) J_{i-1}^{\alpha}\right]\right]=\lim _{\alpha \rightarrow \infty} \mathbb{E}\left[J_{i-1}^{\alpha} \mathbb{E}_{i-1}\left[\left(\pi_{i}+g_{i}\right)\right]\right] \\
& \leq \mathbb{E}\left[\frac{\bar{\rho}_{i-1}}{\pi_{i-1}} \mathbb{E}_{i-1}\left[\pi_{i}+g_{i}\right]\right]=\mathbb{E}\left[\bar{\rho}_{i-1}\right],
\end{aligned}
$$

since

$$
J_{i-1}^{\alpha} \leq \frac{\bar{\rho}_{i-1}}{\pi_{i-1}} .
$$

Thus we see for all $i \geq 1$ that if $\mathbb{E}\left[\bar{\rho}_{i-1}\right]<\infty$ then $\mathbb{E}\left[\bar{\rho}_{i}\right]<\infty$. But $\bar{\rho}_{0}<\infty$ by construction; hence by induction we deduce that $\mathbb{E}\left[\bar{\rho}_{i}\right]<\infty$ for all $i \geq 0$.

To show that $\lim _{j \rightarrow \infty}\left\{\bar{B}_{j}\right\}=\infty$ let us assume the contrary and show that this leads to a contradiction. Suppose, in particular, that there were to exist a number $b$ such that $\bar{B}_{i}<b$ for all $i \in \mathbb{N}_{0}$. Then for all $i \in \mathbb{N}_{0}$ we would have

$$
\mathbb{E}\left[\frac{\bar{\rho}_{i}}{\bar{B}_{i}}\right]>\frac{1}{b} \mathbb{E}\left[\bar{\rho}_{i}\right]=\frac{\bar{\rho}_{0}}{b} .
$$

But by construction we know that $\lim _{i \rightarrow \infty} \mathbb{E}\left[\pi_{i}\right]=0$ and hence

$$
\lim _{i \rightarrow \infty} \mathbb{E}\left[\frac{\bar{\rho}_{i}}{\bar{B}_{i}}\right]=0 \text {. }
$$


Thus given any $\epsilon>0$ we can find a time $t_{i}$ such that

$$
\mathbb{E}\left[\frac{\bar{\rho}_{i}}{\bar{B}_{i}}\right]<\epsilon .
$$

But this is inconsistent with (40); and thus we conclude that $\lim _{j \rightarrow \infty} \bar{B}_{j}=\infty$. That completes the proof of Proposition 4.

IV. Nominal discount bonds. Now we proceed to consider the properties of nominal discount bonds. By such an instrument we mean an asset that pays a single dividend consisting of one unit of domestic currency at some designated time $t_{j}$. For the price $P_{i j}$ at time $t_{i}(i<j)$ of a discount bond that matures at time $t_{j}$ we thus have

$$
P_{i j}=\frac{1}{\pi_{i}} \mathbb{E}_{i}\left[\pi_{j}\right] .
$$

Since $\pi_{i}>0$ for all $i \in \mathbb{N}$, and $\mathbb{E}_{i}\left[\pi_{j}\right]<\pi_{i}$ for all $i<j$, it follows that $0<P_{i j}<1$ for all $i<j$. We observe, in particular, that the associated interest rate $R_{i j}$ defined by

$$
P_{i j}=\frac{1}{1+R_{i j}}
$$

is strictly positive. In our theory we regard a discount bond as a "dividend-paying" asset. Thus in the case of a discount bond with maturity $t_{j}$ we have $P_{j j}=0$ and $D_{j}=1$. Usually discount bonds are defined by setting $P_{j j}=1$ at maturity, with $D_{j}=0$; but it is more logical to regard the bonds as giving rise to a unit cash flow at maturity. The definition of the discount bond system does not involve the specific choice of the positive-return asset.

It is important to point out that in the present framework there is no reason or need to model the dynamics of $\left\{P_{i j}\right\}$, or to model the volatility structure of the discount bonds. Indeed, from the present point of view this would be a little artificial. The important issue, rather, is how to model the pricing kernel. Thus, our scheme differs somewhat in spirit from the discrete-time models discussed, e.g., in Heath et al. 1990, and Filipović \& Zabczyk 2002.

As a simple example of a family of discrete-time interest rate models admitting tractable formulae for the associated discount bond price processes, suppose we set

$$
\pi_{i}=\alpha_{i}+\beta_{i} N_{i}
$$

where $\left\{\alpha_{i}\right\}$ and $\left\{\beta_{i}\right\}$ are strictly-positive, strictly-decreasing deterministic sequences, satisfying $\lim _{i \rightarrow \infty} \alpha_{i}=0$ and $\lim _{i \rightarrow \infty} \beta_{i}=0$, and where $\left\{N_{i}\right\}$ is a strictly positive martingale. Then by (43) we have

$$
P_{i j}=\frac{\alpha_{j}+\beta_{j} N_{i}}{\alpha_{i}+\beta_{i} N_{i}}
$$

thus giving a family of "rational" interest rate models. Note that in a discrete-time setting we can produce classes of models that have no immediate analogues in continuous timefor example, we can let $\left\{N_{i}\right\}$ be the natural martingale associated with a branching process.

Now we shall demonstrate that any discount bond system consistent with our general scheme admits a representation of the Flesaker-Hughston type. For accounts of the 
Flesaker-Hughston theory see, e.g., Flesaker \& Hughston 1996, Rutkowski 1997, Hunt \& Kennedy 2000, or Jin \& Glasserman 2001.

Proposition 5. Let $\left\{\pi_{i}\right\},\left\{\bar{B}_{i}\right\},\left\{P_{i j}\right\}$ satisfy the conditions of Axioms A and B. Then there exists a family of positive martingales $\left\{m_{i n}\right\}_{0 \leq i \leq n}$ indexed by $n \in \mathbb{N}$ such that

$$
P_{i j}=\frac{\sum_{n=j+1}^{\infty} m_{i n}}{\sum_{n=i+1}^{\infty} m_{i n}} .
$$

Proof. We shall use the fact that $\pi_{i}$ can be written in the form

$$
\pi_{i}=\mathbb{E}_{i}\left[G_{\infty}\right]-G_{i}=\mathbb{E}_{i}\left[\sum_{n=1}^{\infty} g_{n}\right]-\sum_{n=1}^{i} g_{n}=\mathbb{E}_{i}\left[\sum_{n=i+1}^{\infty} g_{n}\right],
$$

where $g_{i}=G_{i}-G_{i-1}$ for each $i \geq 1$. Then $g_{i}>0$ for all $i \geq 1$ since $\left\{G_{i}\right\}$ is a strictly increasing sequence. By the monotone convergence theorem we have

$$
\pi_{i}=\sum_{n=i+1}^{\infty} \mathbb{E}_{i}\left[g_{n}\right]
$$

and

$$
\mathbb{E}_{i}\left[\pi_{j}\right]=\sum_{n=j+1}^{\infty} \mathbb{E}_{i}\left[g_{n}\right]
$$

For each $n \geq 1$ we define $m_{i n}=\mathbb{E}_{i}\left[X_{n}\right]$. Then for each $n \in \mathbb{N}$ we see that $\left\{m_{i n}\right\}_{0 \leq i \leq n}$ is a strictly positive martingale, and (47) follows immediately.

V. Nominal money-market account. In the analysis presented so far we have assumed that the positive-return process $\left\{\bar{B}_{i}\right\}$ is $\left\{F_{i}\right\}$-adapted, but is not necessarily previsible. Many of our conclusions are valid under the weaker hypothesis of adaptedness, as we have seen. There are also economic motivations behind the use of the more general assumption. One can imagine that the time sequence $\left\{t_{i}\right\}$ is in reality a "course graining" of a finer time sequence that includes the original sequence as a sub-sequence. Then likewise one can imagine that $\left\{\bar{B}_{i}\right\}$ is a sub-sequence of a finer process that assigns a value to the positive-return asset at each time in the finer time sequence. Finally, we can imagine that $\left\{\mathcal{F}_{i}\right\}$ is a sub-filtration of a finer filtration based on the finer sequence. In the case of a money market account, where the rate of interest is set at the beginning of each short deposit period, we would like to regard the relevant value process as being previsible with respect to the finer filtration, but merely adapted with respect to the course-grained filtration.

Do positive-return assets, other than the standard previsible money market account, actually exist in a discrete-time setting? The following example gives an affirmative answer. In the setting of the standard binomial model, in the case of a single period, let $S_{0}$ denote the value at time 0 of a risky asset, and let $\{U, D\}$ denote its possible values at time 1. Let $B_{0}$ and $B_{1}$ denote the values at times 0 and 1 of a deterministic money-market account. We assume that $B_{1}>B_{0}$ and $U>S_{0} B_{1} / B_{0}>D$. A standard calculation shows that the risk-neutral probabilities for $S_{0} \rightarrow U$ and $S_{0} \rightarrow D$ are given by $p^{*}$ and $1-p^{*}$, where $p^{*}=\left(S_{0} B_{1} / B_{0}-D\right) /(U-D)$. We shall now construct a "positive-return" asset, 
i.e. an asset with initial value $\bar{S}_{0}$ and with possible values $\{\bar{U}, \bar{D}\}$ at time 1 such that $\bar{U}>\bar{S}_{0}$ and $\bar{D}>\bar{S}_{0}$. Risk-neutral valuation implies that $\bar{S}_{0}=\left(B_{0} / B_{1}\right)\left[p^{*} \bar{U}+\left(1-p^{*}\right) \bar{D}\right]$. Thus, given $\bar{S}_{0}$, we can determine $\bar{U}$ in terms of $\bar{D}$. A calculation then shows that if $\left(B_{1} / B_{0}-p^{*}\right) /\left(1-p^{*}\right)>\bar{D} / \bar{S}_{0}>1$, then $\bar{U}>\bar{S}_{0}$ and $\bar{D}>\bar{S}_{0}$, as desired. Thus, in the one-period binomial model, for the given initial value $\bar{S}_{0}$, we obtain a one-parameter family of positive-return assets.

Let us consider now the special case where the positive-return asset is previsible. Thus for $i \geq 1$ we assume that $B_{i}$ is $\mathcal{F}_{i-1}$-measurable and we drop the "bar" over $B_{i}$ to signify the fact that we are now considering a money-market account. In that case we have

$$
P_{i-1, i}=\frac{1}{\pi_{i-1}} \mathbb{E}_{i-1}\left[\pi_{i}\right]=\frac{B_{i-1}}{\rho_{i-1}} \mathbb{E}_{i-1}\left[\frac{\rho_{i}}{B_{i}}\right]=\frac{B_{i-1}}{B_{i}},
$$

by virtue of the martingale property of $\left\{\rho_{i}\right\}$. Thus, in the case of a money-market account we see that

$$
P_{i-1, i}=\frac{1}{1+r_{i}}
$$

where $r_{i}=R_{i-1, i}$. In other words, the rate of return on the money-market account is previsible, and is given by the one-period simple discount factor associated with the discount bond that matures at time $t_{i}$.

Reverting to the general situation, it follows that if we are given a pricing kernel $\left\{\pi_{i}\right\}$ on a probability space $(\Omega, \mathcal{F}, \mathbb{P})$ with filtration $\left\{\mathcal{F}_{i}\right\}$, and a system of assets satisfying Axioms $\mathrm{A}$ and $\mathrm{B}$, then we can construct a plausible candidate for an associated previsible money market account by setting $B_{0}=1$ and defining

$$
B_{i}=\left(1+r_{i}\right)\left(1+r_{i-1}\right) \cdots\left(1+r_{1}\right)
$$

for $i \geq 1$, where

$$
r_{i}=\frac{\pi_{i-1}}{\mathbb{E}_{i-1}\left[\pi_{i}\right]}-1
$$

We shall refer to the process $\left\{B_{i}\right\}$ thus constructed as the "natural" money market account associated with the pricing kernel $\left\{\pi_{i}\right\}$.

To justify this nomenclature, we need to verify that $\left\{B_{i}\right\}$, so constructed, satisfies the conditions of Axioms A and B. To this end, we make note of the following decomposition. Let $\left\{\pi_{i}\right\}$ be a positive supermartingale satisfying $\mathbb{E}_{i}\left[\pi_{j}\right]<\pi_{i}$ for all $i<j$ and $\lim _{j \rightarrow \infty}\left[\pi_{j}\right]=0$. Then as an identity we can write

$$
\pi_{i}=\frac{\rho_{i}}{B_{i}}
$$

where

$$
\rho_{i}=\frac{\pi_{i}}{\mathbb{E}_{i-1}\left[\pi_{i}\right]} \frac{\pi_{i-1}}{\mathbb{E}_{i-2}\left[\pi_{i-1}\right]} \cdots \frac{\pi_{1}}{\mathbb{E}_{0}\left[\pi_{1}\right]} \pi_{0}
$$

for $i \geq 0$, and

$$
B_{i}=\frac{\pi_{i-1}}{\mathbb{E}_{i-1}\left[\pi_{i}\right]} \frac{\pi_{i-2}}{\mathbb{E}_{i-2}\left[\pi_{i-1}\right]} \cdots \frac{\pi_{1}}{\mathbb{E}_{1}\left[\pi_{2}\right]} \frac{\pi_{0}}{\mathbb{E}_{0}\left[\pi_{1}\right]}
$$

for $i \geq 1$, with $B_{0}=1$. Thus, in this scheme we have

$$
\rho_{i}=\frac{\pi_{i}}{\mathbb{E}_{i-1}\left[\pi_{i}\right]} \rho_{i-1}
$$


with the initial condition $\rho_{0}=\pi_{0}$; and

$$
B_{i}=\frac{\pi_{i-1}}{\mathbb{E}_{i-1}\left[\pi_{i}\right]} B_{i-1},
$$

with the initial condition $B_{0}=1$. It is evident that $\left\{\rho_{i}\right\}$ as thus defined is $\left\{\mathcal{F}_{i}\right\}$-adapted, and that $\left\{B_{i}\right\}$ is previsible and strictly increasing. Making use of the identity (59) we are in a position to establish the following:

Proposition 6. Let $\left\{\pi_{i}\right\}$ be a non-negative supermartingale satisfying $\mathbb{E}_{i}\left[\pi_{j}\right]<\pi_{i}$ for all $i<j \in \mathbb{N}_{0}$, and $\lim _{i \rightarrow \infty} \mathbb{E}\left[\pi_{i}\right]=0$. Let $\left\{B_{i}\right\}$ be defined by $B_{0}=1$ and $B_{i}=\prod_{n=1}^{i}\left(1+r_{n}\right)$ for $i \geq 1$, where $1+r_{i}=\pi_{i-1} / \mathbb{E}_{i-1}\left[\pi_{i}\right]$, and set $\rho_{i}=\pi_{i} B_{i}$ for $i \geq 0$. Then $\left\{\rho_{i}\right\}$ is a martingale, and the interest rate system defined by $\left\{\pi_{i}\right\},\left\{B_{i}\right\}$, and $\left\{P_{i j}\right\}$ satisfies Axioms $\mathrm{A}$ and $\mathrm{B}$.

Proof. To show that $\left\{\rho_{i}\right\}$ is a martingale it suffices to verify for all $i \geq 1$ that $\mathbb{E}\left[\rho_{i}\right]<\infty$ and that $\mathbb{E}_{i-1}\left[\rho_{i}\right]=\rho_{i-1}$. In particular, if $\mathbb{E}\left[\rho_{i}\right]<\infty$ then the "take out what is known rule" is applicable, and by (58) we have

$$
\mathbb{E}_{i-1}\left[\rho_{i}\right]=\mathbb{E}_{i-1}\left[\frac{\pi_{i}}{\mathbb{E}_{i-1}\left[\pi_{i}\right]} \rho_{i-1}\right]=\rho_{i-1} .
$$

Thus to show that $\left\{\rho_{i}\right\}$ is a martingale it remains to verify that $\mathbb{E}\left[\rho_{i}\right]<\infty$. Let us write

$$
J_{i-1}^{\alpha}=\min \left[\frac{\rho_{i-1}}{\mathbb{E}_{i-1}\left[\pi_{i}\right]}, \alpha\right]
$$

for $\alpha \in \mathbb{N}_{0}$. Then by monotone convergence and the tower property we have

$$
\mathbb{E}\left[\rho_{i}\right]=\mathbb{E}\left[\pi_{i} \lim _{\alpha \rightarrow \infty} J_{i-1}^{\alpha}\right]=\lim _{\alpha \rightarrow \infty} \mathbb{E}\left[\pi_{i} J_{i-1}^{\alpha}\right]=\lim _{\alpha \rightarrow \infty} \mathbb{E}\left[\mathbb{E}_{i-1}\left[\pi_{i} J_{i-1}^{\alpha}\right]\right] .
$$

But since the variable $J_{i-1}^{\alpha}$ is bounded we can move it outside the inner conditional expectation to give

$$
\mathbb{E}\left[\rho_{i}\right]=\lim _{\alpha \rightarrow \infty} \mathbb{E}\left[J_{i-1}^{\alpha} \mathbb{E}_{1-i}\left[\pi_{i}\right]\right] \leq \mathbb{E}\left[\rho_{i-1}\right]
$$

since

$$
J_{i-1}^{\alpha} \leq \frac{\rho_{i-1}}{\mathbb{E}_{i-1}\left[\pi_{i}\right]}
$$

Thus we see for all $i \geq 1$ that if $\mathbb{E}\left[\rho_{i-1}\right]<\infty$ then $\mathbb{E}\left[\rho_{i}\right]<\infty$. But $\rho_{0}<\infty$ by construction, and hence by induction we deduce that $\mathbb{E}\left[\rho_{i}\right]<\infty$ for all $i \geq 0$.

The martingale $\left\{\rho_{i}\right\}$ is the likelihood ratio process appropriate for a change of measure from the objective measure $\mathbb{P}$ to the equivalent martingale measure $\mathbb{Q}$ characterised by the property that non-dividend-paying assets are martingales when expressed in units of the money-market account. An interesting feature of Proposition 6 is that no integrability condition is required on $\left\{\rho_{i}\right\}$. In other words, the natural previsible money market account defined by (57) "automatically" satisfies the conditions of Axiom A. For some purposes it may therefore be advantageous to incorporate the existence of the natural money market account directly into the axioms. Then instead of Axiom B we would have:

Axiom B*. There exists a strictly-positive non-dividend paying asset, the money-market account, with value process $\left\{B_{i}\right\}_{i \geq 0}$, having the properties that $B_{i+1}>B_{i}$ for all $i \in \mathbb{N}_{0}$ 
and that $B_{i}$ is $\mathcal{F}_{i-1}$-measurable for all $i \in \mathbb{N}$. We assume that $\left\{B_{i}\right\}$ is unbounded in the sense that for any $b \in \mathbb{R}$ there exists a time $t_{i}$ such that $B_{i}>b$.

The content of Proposition 6 is that Axioms A and B together imply Axiom B*. As an exercise we shall establish that the class of interest rate models satisfying Axioms A and $\mathrm{B}^{*}$ is non-vacuus. In particular, suppose we consider the "rational" models defined by equations (45) and (46) for some choice of the martingale $\left\{N_{i}\right\}$. It is straightforward to see that the unique previsible money market account in this model is given by $B_{0}=1$ and

$$
B_{i}=\prod_{n=1}^{i} \frac{\alpha_{n-1}+\beta_{n-1} N_{n-1}}{\alpha_{n}+\beta_{n} N_{n-1}}
$$

for $i \geq 1$. For $\left\{\rho_{i}\right\}$ we then have

$$
\rho_{i}=\rho_{0} \prod_{n=1}^{i} \frac{\alpha_{n}+\beta_{n} N_{n}}{\alpha_{n}+\beta_{n} N_{n-1}},
$$

where $\rho_{0}=\alpha_{0}+\beta_{0} N_{0}$. But it is easy to check that for each $i \geq 0$ the random variable $\rho_{i}$ is bounded; therefore $\left\{\rho_{i}\right\}$ is a martingale, and the money market account process $\left\{B_{i}\right\}$ satisfies the conditions of Axioms A and $\mathrm{B}^{*}$.

Now let us return to the Doob decomposition for $\left\{\pi_{i}\right\}$ given in formula (4). Evidently, we have $\pi_{i}=\mathbb{E}_{i}\left[A_{\infty}\right]-A_{i}$, with

$$
A_{i}=\sum_{n=0}^{i-1}\left(\pi_{n}-\mathbb{E}_{n}\left[\pi_{n+1}\right]\right)=\sum_{n=0}^{i-1} \pi_{n}\left(1-\frac{\mathbb{E}_{n}\left[\pi_{n+1}\right]}{\pi_{n}}\right)=\sum_{n=0}^{i-1} \pi_{n} r_{n+1} P_{n, n+1},
$$

where $\left\{r_{i}\right\}$ is the previsible short rate process defined by (52). The pricing kernel can therefore be put in the form

$$
\pi_{i}=\mathbb{E}_{i}\left[\sum_{n=i}^{\infty} \pi_{n} r_{n+1} P_{n, n+1}\right]
$$

Comparing the Doob decomposition (68) with the alternative decomposition given by (31), we deduce that if we set

$$
\bar{r}_{i}=\frac{r_{i} \pi_{i-1} P_{i-1, i}}{\pi_{i}}
$$

then we obtain a positive-return asset for which the corresponding decomposition of the pricing kernel, as given by (31), is the Doob decomposition. On the other hand, since the money-market account is a positive-return asset, by Proposition 3 we can also write

$$
\pi_{i}=\mathbb{E}_{i}\left[\sum_{n=i+1}^{\infty} \pi_{n} r_{n}\right]
$$

As a consequence, we see that the price process of a pure dividend asset can be written in the symmetrical form

$$
S_{i}=\frac{\mathbb{E}_{i}\left[\sum_{n=i+1}^{\infty} \pi_{n} D_{n}\right]}{\mathbb{E}_{i}\left[\sum_{n=i+1}^{\infty} \pi_{n} r_{n}\right]},
$$

where $\left\{D_{n}\right\}$ is the dividend process, and $\left\{r_{n}\right\}$ is the short rate process. 
VI. Information-based interest rate models. So far in the discussion we have regarded the pricing kernel $\left\{\pi_{i}\right\}$ and the filtration $\left\{\mathcal{F}_{i}\right\}$ as being exogenously specified. To develop the framework further we need to make a more specific indication of how the pricing kernel is determined, and how information is made available to market participants. To obtain a realistic model for $\left\{\pi_{i}\right\}$ we need to develop the model in conjunction with a theory of consumption, money supply, price level, inflation, real interest rates, and information. We shall proceed in two steps. First we consider a general "reduced-form" model for nominal interest rates, in which we model the filtration explicitly; then in the next section we consider a more general "structural" model in which both the nominal and the real interest rate systems are determined.

Our reduced-form model for interest rates is based on the theory of $X$-factors, following Brody et al. 2008 and Macrina 2006. Associated with each $t_{i}$ we introduce a collection of random variables $X_{i}^{\alpha}\left(\alpha=1, \ldots, m_{i}\right)$, where $m_{i}$ denotes the number of random variables associated with $t_{i}$. For each $n$, we assume that the random variables $X_{1}^{\alpha}, X_{2}^{\alpha}, \ldots, X_{n}^{\alpha}$ are independent. We regard $X_{n}^{\alpha}$ as being "revealed" at time $t_{n}$, and hence $\mathcal{F}_{n}$-measurable. More precisely, we shall construct the filtration $\left\{\mathcal{F}_{i}\right\}$ in such a way that this property holds. Intuitively, we can think of $X_{1}^{\alpha}, X_{2}^{\alpha}, \ldots, X_{n}^{\alpha}$ as being the independent macroeconomic "market factors" that determine cash flows at $t_{n}$.

Let us consider how the filtration will be modelled. For each $j \in \mathbb{N}_{0}$, at any time $t_{i}$ before $t_{j}$ only partial information about the market factors $X_{j}^{\alpha}$ will be available to market participants. We model this partial information for each market factor $X_{j}^{\alpha}$ by defining a discrete-time information process $\left\{\xi_{t_{i} t_{j}}^{\alpha}\right\}_{0 \leq t_{i} \leq t_{j}}$, setting

$$
\xi_{t_{i} t_{j}}^{\alpha}=\sigma t_{i} X_{j}^{\alpha}+\beta_{t_{i} t_{j}}^{\alpha} .
$$

Here $\left\{\beta_{t_{i} t_{j}}^{\alpha}\right\}_{0 \leq t_{i} \leq t_{j}}$ can, for each value of $\alpha$, be thought of as an independent discretised Brownian bridge. Thus, we consider a standard Brownian motion starting at time zero and ending at time $t_{j}$, and sample its values at the times $\left\{t_{i}\right\}_{i=0, \ldots, j}$. Let us write $\xi_{i j}^{\alpha}=$ $\xi_{t_{i} t_{j}}^{\alpha}$ and $\beta_{i j}^{\alpha}=\beta_{t_{i} t_{j}}^{\alpha}$, in keeping with our usual shorthand conventions for discrete-time modelling. For each value of $\alpha$ we have $\mathbb{E}\left[\beta_{i j}^{\alpha}\right]=0$ and

$$
\operatorname{Cov}\left[\beta_{i k}^{\alpha}, \beta_{j k}^{\alpha}\right]=\frac{t_{i}\left(t_{k}-t_{j}\right)}{t_{k}}
$$

for $i \leq j \leq k$. We assume that the bridge processes are independent of the $X$-factors (i.e., the macroeconomic factors); and hence that the information processes are independent of one another. Finally, we assume that the market filtration is generated collectively by the information processes. For each value of $k$ the sigma-algebra $\mathcal{F}_{k}$ is generated by the random variables $\left\{\xi_{i j}^{\alpha}\right\}_{0 \leq i \leq j \leq k}$.

Thus, the filtration is not simply "given", but rather is modelled explicitly. It is straightforward to verify that for each value of $\alpha$ the process $\left\{\xi_{i j}^{\alpha}\right\}$ has the Markov property. The proof follows the pattern of the continuous-time argument. This has the implication that the conditional expectation of a function of the market factors $X_{j}^{\alpha}$, taken with respect to $\mathcal{F}_{i}$, can be reduced to a conditional expectation with respect to the sigma-algebra $\sigma\left(\xi_{i j}^{\alpha}\right)$. Thus, the history of the process $\left\{\xi_{n j}^{\alpha}\right\}_{n=0,1, \ldots, i}$ can be neglected, and only the most "recent" information, $\xi_{i j}^{\alpha}$, needs to be considered in taking the con- 
ditional expectation. For example, in the case of a function of a single $\mathcal{F}_{j}$-measurable market factor $X_{j}$, with the associated information process $\left\{\xi_{n j}\right\}_{n=0,1, \ldots, j}$, we obtain:

$$
\mathbb{E}\left[f\left(X_{j}\right) \mid \mathcal{F}_{i}\right]=\frac{\int_{0}^{\infty} p(x) f(x) \exp \left[\frac{t_{j}}{t_{j}-t_{i}}\left(\sigma x \xi_{i j}-\frac{1}{2} \sigma^{2} x^{2} t_{i}\right)\right] \mathrm{d} x}{\int_{0}^{\infty} p(x) \exp \left[\frac{t_{j}}{t_{j}-t_{i}}\left(\sigma x \xi_{i j}-\frac{1}{2} \sigma^{2} x^{2} t_{i}\right)\right] \mathrm{d} x},
$$

for $i \leq j$, where $p(x)$ denotes the a priori density for $X_{j}$. In the formula above we have presented the case of a continuous random variable taking non-negative values; the extension to other classes of random variables is straightforward.

Now we are in a position to state how we propose to model the pricing kernel. First, we shall assume that $\left\{\pi_{i}\right\}$ is adapted to the market filtration $\left\{\mathcal{F}_{i}\right\}$. This is clearly a natural assumption from an economic point of view, and is necessary for the general consistency of the theory. This means that the random variable $\pi_{j}$, for any fixed value of $j$, can be expressed as a function of the totality of the available market information at time $j$. In other words, $\pi_{j}$ is a function of the values taken, between times 0 and $j$, of the information processes associated with the various market factors.

Next we make the assumption that $\pi_{j}$ (for any fixed $j$ ) depends on the values of only a finite number of information processes. This corresponds to the intuitive idea that when we price a contingent claim, there is a limit to the amount of information we can consider.

But this implies that expectations of the form $\mathbb{E}_{i}\left[\pi_{j}\right]$, for $i \leq j$, can be computed explicitly. Since $\pi_{j}$ can be expressed as a function of a collection of intertemporal information variables, the relevant conditional expectations can be worked out in closed form. As a consequence, we are led to a system of tractable expressions for the discount bond prices and the previsible money market account. We are left only with the question of what is the correct functional form for $\left\{\pi_{i}\right\}$, given the relevant market factors. If we simply "postulate" a form for $\left\{\pi_{i}\right\}$, then we say that we have a "reduced-form" model. If we provide an economic argument that leads to a specific form for $\left\{\pi_{i}\right\}$, then we say that we have a "structural" model.

VII. Models for inflation and index-linked securities. For a more complete picture we must regard the nominal interest rate system as embedded in a larger system that takes into account the macroeconomic factors that inter-relate the money supply, aggregate consumption, and the price level. We shall present a simple model in this spirit that is consistent with the information-based approach.

To this end we introduce the following quantities. We envisage a closed economy with aggregate consumption $\left\{k_{i}\right\}_{i \geq 1}$. Consumption takes place at discrete times, and $k_{i}$ denotes the aggregate level of consumption, in units of goods and services, taking place at time $t_{i}$. We write $\left\{M_{i}\right\}_{i \geq 0}$ for the process corresponding to the nominal money supply, and $\left\{C_{i}\right\}_{i \geq 0}$ for the process of the consumer price index (the "price level"). For convenience we can regard $\left\{k_{i}\right\}$ and $\left\{M_{i}\right\}$ as being expressed on a per capita basis. Hence these quantities can be regarded, respectively, as the consumption and money balance associated with a representative agent.

We assume that at each $t_{i}$ the agent receives a benefit from the money balance maintained in the economy; this is given in nominal terms by $\lambda_{i} M_{i}$, where $\lambda_{i}$ is the nominal 
liquidity benefit received by the agent per unit of money "carried" by the agent, and $M_{i}$ is the money supply, expressed on a per capita basis, at that time. The corresponding "real" benefit (in units of goods and services) provided to the agent by the money supply at time $t_{i}$ is defined by the expression

$$
l_{i}=\frac{\lambda_{i} M_{i}}{C_{i}} .
$$

It follows that we can think of $\left\{\lambda_{i}\right\}$ as a kind of "convenience yield" process associated with the money supply. Rather in the way a country will obtain a convenience yield (per barrel) from its oil reserves, which can be expressed on a per capita basis, likewise an economy derives a convenience yield (per unit of money) from its money supply. It is important to note that what matters is the real benefit of the money supply, which can be thought of effectively as a flow of goods and services emanating from the presence of the money supply. It is possible that the "wealth" attributable to the face value of the money may in totality be insignificant. For example, if the money supply consists of notes issued by the government, and hence takes the form of government debt, then the per capita wealth associated with the face value of the notes is null, since the representative agent is also responsible (ultimately) for a share of the government debt. Nevertheless, the presence of the money supply confers an overall positive flow of benefit to the agent. If the money supply consists, say, of gold coins, or units of some other valuable commodity, then the face value of the money supply will make a positive contribution to overall wealth, as well as providing a liquidity benefit.

Our goal is to obtain a consistent structural model for the pricing kernel $\left\{\pi_{i}\right\}_{i \geq 0}$. We assume that the representative agent gets utility both from consumption and from the real benefit of the money supply in the spirit of Sidrauski 1969. Let $U(x, y)$ be a standard bivariate utility function $U: \mathbb{R}^{+} \times \mathbb{R}^{+} \rightarrow \mathbb{R}$, satisfying $U_{x}>0, U_{y}>0, U_{x x}<0, U_{y y}<0$, and $U_{x x} U_{y y}>\left(U_{x y}\right)^{2}$. Then the objective of the representative agent is to maximise an expression of the form

$$
J=\mathbb{E}\left[\sum_{n=0}^{N} \mathrm{e}^{-\gamma t_{n}} U\left(k_{n}, l_{n}\right)\right]
$$

over the time horizon $\left[t_{0}, t_{1}, \ldots, t_{N}\right]$, where $\gamma$ is the appropriate discount rate applicable to delayed gains in utility. For simplicity of exposition we assume a constant discount rate. The optimisation problem faced by the agent is subject to the budget constraint

$$
W=\mathbb{E}\left[\sum_{n=0}^{N} \pi_{n}\left(C_{n} k_{n}+\lambda_{n} M_{n}\right)\right] .
$$

Here $W$ represents the total per capita wealth, in nominal terms, available for consumption related expenditure over the given time horizon. The agent can maintain a position in money, and "consume" the benefit of the money; or the money position can be liquidated (in part, or in whole) to purchase consumption goods. In any case, we must include the value of the benefit of the money supply in the budget. Since the presence of the money supply "adds value", we need to recognise this value as a constituent of the budget. The budget includes also any net initial funds available, together with the value of expected income (e.g., derivable from labour or natural resources) over the relevant period. 
The fact that the utility depends on the real benefit of the money supply, whereas the budget depends on the nominal value of the money supply, leads to a fundamental relationship between the processes $\left\{k_{i}\right\},\left\{M_{i}\right\},\left\{C_{i}\right\}$, and $\left\{\lambda_{i}\right\}$. Introducing a Lagrange multiplier $\mu$, after some re-arrangement we obtain the associated unconstrained optimisation problem, for which the objective is to maximise the following expression:

$$
\mathbb{E}\left[\sum_{n=0}^{N} \mathrm{e}^{-\gamma t_{n}} U\left(k_{n}, l_{n}\right)-\mu \sum_{n=0}^{N} \pi_{n} C_{n}\left(k_{n}+l_{n}\right)\right] .
$$

A straightforward argument then shows that the solution for the optimal policy (if it exists) satisfies the first order conditions

$$
U_{x}\left(k_{n}, l_{n}\right)=\mu \mathrm{e}^{\gamma t_{n}} \pi_{n} C_{n},
$$

and

$$
U_{y}\left(k_{n}, l_{n}\right)=\mu \mathrm{e}^{\gamma t_{n}} \pi_{n} C_{n},
$$

for each value of $n$ in the relevant time frame, where $\mu$ is determined by the budget constraint. As a consequence we obtain the fundamental relation

$$
U_{x}\left(k_{n}, \lambda_{n} M_{n} / C_{n}\right)=U_{y}\left(k_{n}, \lambda_{n} M_{n} / C_{n}\right),
$$

which allows us to eliminate any one of the variables $k_{n}, M_{n}, \lambda_{n}$, and $C_{n}$ in terms of the other three. In this way, for a given level of consumption, money supply, and liquidity benefit, we can work out the associated price level. Then by use of (79), or equivalently (80), we can deduce the form taken by the nominal pricing kernel, and hence the corresponding interest rate system. We also obtain thereby an expression for the "real" pricing kernel $\left\{\pi_{i} C_{i}\right\}$.

We shall take the view that aggregate consumption, the liquidity benefit rate, and the money supply level are all determined exogenously. In particular, in the informationbased framework we take these processes to be adapted to the market filtration, and hence determined, at any given time, by the values of the information variables upon which they depend. The theory outlined above then shows how the values of the real and nominal pricing kernels can be obtained, at each time, as functions of the relevant information variables.

It will be useful to have an explicit example in mind, so let us consider a standard "log-separable" utility function of the form

$$
U(x, y)=A \ln (x)+B \ln (y),
$$

where $A$ and $B$ are non-negative constants. From the fundamental relation (81) we immediately obtain

$$
\frac{A}{k_{n}}=\frac{B}{l_{n}}
$$

and hence the equality

$$
k_{n} C_{n}=\frac{A}{B} \lambda_{n} M_{n}
$$

Thus, in the case of log-separable utility we see that the level of consumption, in nominal terms, is always given by a fixed proportion of the nominal liquidity benefit obtained 
from the money supply. For fixed values of $\lambda_{n}$ and $k_{n}$, we note, for example, that an increase in the money supply leads to an increase in the price level.

One observes that in the present framework we derive an expression for the consumer price index process. This contrasts with current well-known methodologies for pricing inflation-linked securities (see, e.g., Hughston 1998, Jarrow \& Yildirim 2003) where the form of the consumer price index is specified on an exogenous basis.

The quantity $k_{n} C_{n} / M_{n}$ is commonly referred to as the "velocity" of money. It measures, roughly speaking, the rate at which money changes hands, as a percentage of the total money supply, as a consequence of consumption. Evidently, in the case of a log-separable utility (82), the velocity has a fixed ratio to the liquidity benefit. This is a satisfying conclusion, which shows that even with a relatively simple assumption about the nature of the utility we are able to obtain an intuitively natural relation between the velocity of money and the liquidity benefit. In particular, if liquidity is increased, then a lower money supply will be required to sustain a given level of nominal consumption, and hence the velocity will be increased as well. The situation when the velocity is constant leads to the so-called "quantity" theory of money, which in the present approach arises in the case of a representative agent with log-separable utility and a constant liquidity benefit.

It is interesting to note that the results mentioned so far, in connection with logseparable utility, are not too sensitive to the choice of the discount rate $\gamma$, which does not enter into the fundamental relation (81). On the other hand, $\gamma$ does enter into the expression for the nominal pricing kernel; in particular, in the log-separable case we obtain the following expression for the pricing kernel:

$$
\pi_{n}=\frac{B \mathrm{e}^{-\gamma t_{n}}}{\mu \lambda_{n} M_{n}} .
$$

Hence, in the log-separable utility theory we can see explicitly the relation between the nominal money supply and the term structure of interest rates.

Consider now a contingent claim with the random nominal payoff $H_{j}$ at $t_{j}$. The value of the claim at $t_{0}$ in the log-separable utility model is given by the following formula:

$$
H_{0}=\lambda_{0} M_{0} \mathrm{e}^{-\gamma t_{j}} \mathbb{E}\left[\frac{H_{j}}{\lambda_{j} M_{j}}\right] .
$$

One sees two different influences on the value of $H_{0}$. First one has the discount factor; but equally one sees the effect of the money supply. For a given level of the liquidity benefit (i.e., for constant $\lambda_{j}$ ), an increase in the likely money supply at time $t_{j}$ will reduce the value of $H_{0}$. This example illustrates how market perceptions of the direction of future monetary policy can affect the valuation of contingent claims. In particular, the value of the money supply $M_{j}$ at $t_{j}$ will be given as a function of the best available information at that time concerning future random factors affecting the economy. The question of how best to model the money supply process $\left\{M_{i}\right\}$ takes us outside of the realm of pure mathematical finance, and into the territory of macroeconomics and, ultimately, political economics. It is interesting to note therefore that an increase in the liquidity benefit rate has the same practical effect on present valuations as an increase in the money supply itself. 
A striking feature of the separable log-utility example is that the consumption process does not enter into the valuation formulae for financial claims. In that case, therefore, one can argue that inflation is a purely monetary phenomenon, insofar as expectations affect present valuations. But for more general utility functions this is not the case. Let us consider, for example, the case of a separable power utility function, writing

$$
U(x, y)=\frac{A}{p} x^{p}+\frac{B}{q} y^{q},
$$

where $p, q \in(-\infty, 1) \backslash\{0\}$. Then a calculation analogous to the previous one shows that the consumer price index is given in this situation by

$$
C_{n}=\left(\frac{A}{B}\right)^{1 /(1-q)} \frac{\lambda_{n} M_{n}}{k_{n}^{(1-p) /(1-q)}} .
$$

Thus in the case of power utility the dependence of the price index on consumption, although always an inverse relation, depends, on the ratio of the coefficients of relative risk aversion associated with real consumption and the money supply benefit. The corresponding expression for the nominal pricing kernel is

$$
\pi_{n}=\frac{B^{\frac{1}{1-q}}}{A^{\frac{q}{1-q}}} \frac{\mathrm{e}^{-\gamma t_{n}} k_{n}^{\frac{q}{1-q}(1-p)}}{\mu \lambda_{n} M_{n}} .
$$

It follows that the value $H_{0}$ at time $t_{0}$ of a contingent claim with the random payoff $H_{j}$ at time $t_{j}$ is given by the following formula:

$$
H_{0}=\frac{\lambda_{0} M_{0}}{k_{0}^{q(1-p) /(1-q)}} \mathrm{e}^{-\gamma t_{j}} \mathbb{E}\left[\frac{H_{j} k_{j}^{q(1-p) /(1-q)}}{\lambda_{j} M_{j}}\right] .
$$

In this situation we see that the valuation depends not only on expectations concerning the money supply, but also on the level of consumption.

Acknowledgements. LPH and AM acknowledge support from EPSRC grant number GR/S22998/01; AM thanks the Public Education Authority of the Canton of Bern, Switzerland, and the UK Universities ORS scheme, for support. We are grateful to T. Björk, D. Brody, I. Buckley, H. Bühlmann, H. Geman, M. Hinnerich, T. Ohmoto, J. Vega, and M. Zervos for helpful ideas and discussions. We thank participants at the ETH Risk Day 2006 Zürich meeting and the May 2007 AMaMeF meeting, Będlewo, Poland for useful comments. This work was carried out in part while LPH was based at the Department of Mathematics, King's College London, and at Perimeter Institute for Theoretical Physics, Waterloo, Ontario, Canada.

\section{References}

D. C. Brody \& L. P. Hughston (2004), Chaos and coherence: a new framework for interest-rate modelling, Proceedings of the Royal Society A460, 85-110.

D. C. Brody, L. P. Hughston \& A. Macrina (2007), Beyond hazard rates: a new framework for credit-risk modelling, in: Advances in Mathematical Finance, Festschrift Volume in Honour of Dilip Madan, ed. by M. Fu, R. Jarrow, J. Yen \& R. Elliott, Birkhäuser, Basel, and Springer, Berlin. 
D. C. Brody, L. P. Hughston \& A. Macrina (2008), Information-based asset pricing, International Journal of Theoretical and Applied Finance 11, 107-142.

J. H. Cochrane (2005), Asset Pricing, Princeton University Press, Princeton.

B. Flesaker \& L. P. Hughston (1996), Positive interest, Risk 9, 46-49.

D. Filipović \& J. Zabczyk (2002), Markovian term structure models in discrete time, Annals of Applied Probability 12, 710-729.

D. Heath, R. Jarrow \& A. Morton (1990), Bond pricing and the term structure of interest rates: a discrete time approximation, Journal of Finance and Quantitative Analysis 25, 419-440.

T. S. Y. Ho \& S. B. Lee (1986), Term-structure movements and pricing interest rate contingent claims, Journal of Finance and Quantitative Analysis 41, 1011-1029.

L. P. Hughston (1998), Inflation derivatives, Merrill Lynch and King's College London report, with added note (2004), downloadable at www.mth.ac.uk.

L. P. Hughston \& A. Rafailidis (2005), A chaotic approach to interest rate modelling, Finance and Stochastics 9, 43-65.

P. J. Hunt \& J. E. Kennedy (2000), Financial Derivatives in Theory and Practice, Wiley, Chichester.

R. Jarrow \& Y. Yildirim (2003), Pricing treasury inflation protected securities and related derivative securities using an HJM model, Journal of Financial and Quantitative Analysis 38, 409.

Y. Jin \& P. Glasserman (2001), Equilibrium positive interest rates: a unified view, Review of Financial Studies 14, 187-214.

A. Macrina (2006), An information-based framework for asset pricing: X-factor theory and its applications, $\mathrm{PhD}$ thesis, King's College London.

F. Mercurio (2005), Pricing inflation-indexed derivatives, Journal of Quantitative Finance 5, 289-302.

P. A. Meyer (1966), Probability and Potentials, Blaisdell, Waltham.

S. R. Pliska (1997), Introduction to Mathematical Finance, Blackwell, Oxford.

L. C. G. Rogers (1997), The potential approach to the term structure of interest rate and foreign exchange rates, Mathematical Finance 7, 157-176.

M. Rutkowski (1997), A note on the Flesaker-Hughston model of the term structure of interest rates, Applied Mathematical Finance 4, 151-163.

S. E. Shreve (2004), Stochastic Calculus for Finance I: the Binomial Asset Pricing Model, Springer, Berlin.

M. Sidrauski (1967), Rational choice and patterns of growth in a monetary economy, American Economic Review 2, 534-544.

J. M. Steele (2001), Stochastic Calculus and Financial Applications, Springer, Berlin.

D. Williams (1991), Probability with Martingales, Cambridge University Press, Cambridge. 